\title{
Atypical Presentation of Sinonasal Cellular Schwannoma: A Nonsolitary Mass with Osseous, Orbital, and Intracranial Invasion
}

\author{
John Gencarelli ${ }^{1}$ Ryan Rourke Rr,2 $^{1,2}$ Tracey Ross ${ }^{1,2}$ Denis H. Gravel ${ }^{2,3}$ Bibianna Purgina ${ }^{2,3}$ \\ David Jordan ${ }^{2,4}$ Charles Agbi ${ }^{2,5}$ Shaun J. Kilty ${ }^{1,2,6}$
}

${ }^{1}$ Department of Otolaryngology - Head and Neck Surgery, University of Ottawa, Ottawa, Ontario, Canada

2 The Ottawa Hospital, Ottawa, Ontario, Canada

${ }^{3}$ Department of Pathology and Laboratory Medicine, University of Ottawa, Ottawa, Ontario, Canada

${ }^{4}$ Department of Ophthalmology, University of Ottawa, Ottawa, Ontario, Canada

${ }^{5}$ Division of Neurosurgery, University of Ottawa, Ottawa, Ontario, Canada

6 The Ottawa Hospital Research Institute (OHRI), Ottawa, Ontario, Canada

\begin{abstract}
Address for correspondence Dr. Shaun Kilty, MD, Department of Otolaryngology - Head and Neck Surgery, Ottawa Hospital, Civic Campus, Parkdale Clinic, Room 459, 737 Parkdale Ave., Ottawa, ON, Canada (e-mail: kiltysj@gmail.com).
\end{abstract}

J Neurol Surg Rep 2014;75:e144-e148.

\begin{abstract}
Objective Sinonasal cellular schwannoma represents $<4 \%$ of head and neck schwannomas. These benign tumors are typically confined to the nasal cavity or ethmoid sinus. We describe an atypical case of sinonasal cellular schwannoma with diffuse paranasal sinus involvement and both intraorbital and intracranial extension.

Results A 62-year-old woman presented with a 6-month history of right orbital proptosis and right-sided headache. Subsequent imaging revealed an invasive paranasal sinus mass extending through the skull base and displacing the right orbit. Preoperative biopsies were not diagnostic but revealed a spindle cell lesion suspicious for malignancy based on lack of encapsulation, infiltration of the sinonasal submucosa, and osseous

Keywords

- cellular schwannoma

- nasal cavity

- sinuses

- paranasal

- intracranial invasion. The patient underwent open skull base surgery, and pathology confirmed a S100-positive nonencapsulated cellular schwannoma.

Conclusion An atypical case of sinonasal cellular schwannoma with intracranial extension is reported. Its presentation is contrary to the common view that these are isolated solitary lesions of the nasoethmoid region. We suggest that sinonasal cellular schwannoma be considered in the differential diagnosis of a poorly defined invasive paranasal sinus mass, particularly following biopsy.
\end{abstract}

\section{Background}

Schwannoma is a benign tumor originating from the Schwann cell of the neural sheath, and 25 to $45 \%$ occur in the head and neck. ${ }^{1}$ Although rare, these tumors may originate in the sinonasal tract. Cellular schwannomas of this area represent
$<4 \%$ of head and neck schwannomas; only an estimated 100 cases of this type have been reported in the literature.,3

Sinonasal schwannomas cause a variety of clinical symptoms that depend on the location and size of the mass. ${ }^{4}$ Patients often present quite late because there is significant room for the tumor to grow in the air-filled sinonasal tract. ${ }^{4}$ received

October 22, 2013

accepted after revision

April 3, 2014

published online

June 24, 2014
DOI http://dx.doi.org/

10.1055/s-0034-1376424. ISSN 2193-6358. (c) 2014 Georg Thieme Verlag KG Stuttgart · New York
License terms

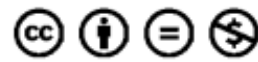


Published descriptions of the radiologic appearance of these benign tumors indicate that they present as a well-demarcated solitary soft tissue mass, most commonly in the nasal cavity or ethmoid sinus. ${ }^{4,5}$ Over time, constant pressure by the mass on the surrounding structures may lead to bone remodeling and erosion. ${ }^{6}$

Cellular schwannoma was first described in 1981 is an uncommon but well-recognized variant of schwannoma. ${ }^{7-12}$ The most common sites of occurrence include the posterior mediastinum and the retroperitoneum. Like conventional schwannomas, these are typically, but not always, encapsulated. Unlike conventional schwannomas, cellular schwannomas demonstrate worrisome clinical and histologic features including bone erosion and both increased cellularity and mitotic activity. Prior to the recognition of this subtype of schwannoma, approximately a third of cellular schwannomas were diagnosed as malignant based on these aggressive features. $^{7-11}$

We describe the presentation, radiologic findings, and treatment of a patient with a sinonasal cellular schwannoma and its aggressive clinical and histologic features. The patient presented with a poorly demarcated diffuse tumor infiltrating all of the ipsilateral paranasal sinuses with concomitant intracranial and orbital extension. Details of the initial presentation, diagnostic assessment, and treatment are highlighted here.

\section{Case Report}

A 62-year-old woman presented with a 6-month history of right orbital proptosis and right-sided headache. She denied diplopia or any change in vision. The headache was described as circumferential and constant, without any positional association or aura. Past medical history included bilateral retinitis pigmentosa, hip replacement, and tubal ligation. She was not taking any medications, and there was no history of smoking or relevant occupational exposures.
Clinical evaluation revealed a 5-mm proptosis and a 6- $\mathrm{mm}$ outward and a 2-mm inferior displacement of the right eye. There was slight under action with both upward and downward gaze. Visual acuity was recorded as 20/400 bilaterally, related to the retinitis pigmentosa history. The remainder of the cranial nerve examination was normal. Examination of the oral cavity and neck was unremarkable. Intranasally, anterior rhinoscopy revealed a fleshy mass originating from the right middle meatus surrounded by purulent secretions. This was substantiated with nasal endoscopy. Cranial computed tomography and magnetic resonance imaging demonstrated an invasive right paranasal sinus mass with intracranial and orbital extension ( - Fig. 1). There was dural involvement.

An initial biopsy revealed a highly cellular spindle cell lesion, likely of peripheral nerve sheath origin, with normal mitotic rate and no vascular invasion. A definitive diagnosis could not be made, but suspicion for malignancy was relatively high based on the lack of encapsulation, infiltration of the sinonasal submucosa, and invasion into surrounding bone and tissue. A second biopsy revealed the same findings. At the first clinical visit, the patient was placed on a 14-day course of amoxicillin-clavulanic acid given the purulent discharge, which completely relieved the headache and resolved the purulent discharge.

The patient underwent an open skull base procedure, and the mass was resected. This involved a bifrontal craniotomy, bilateral tarsorrhaphy, right lateral rhinotomy, partial maxillectomy, sphenoidectomy, posterior nasal septectomy, right dacryocystorhinostomy, right orbital roof and anterior cranial floor resection followed by right orbital roof reconstruction with a split calvarial graft, and a bilateral pericranial flap for closure of the anterior cranial floor (-Fig. 2). The patient was discharged home 1 week following surgery without event. Following surgery, the patient received postoperative radiation therapy due to the extent of invasion and aggressive nature of the tumor, as well as close intracranial margins.
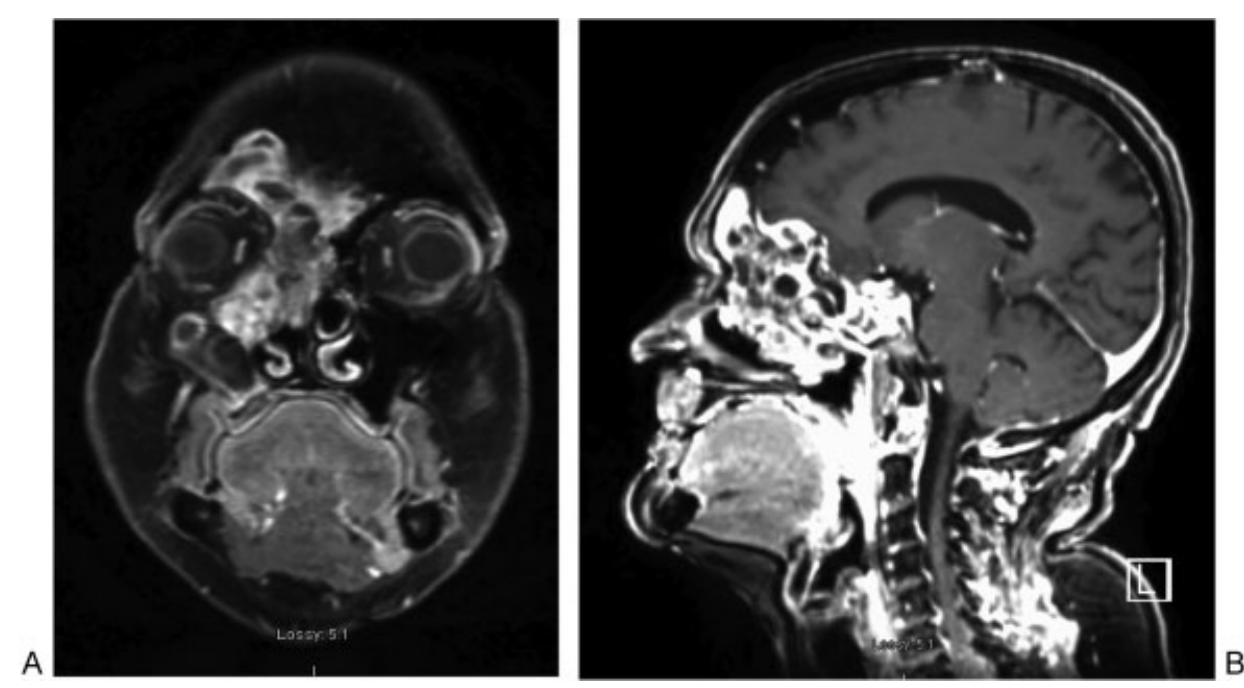

Fig. 1 Magnetic resonance imaging (MRI) demonstrating the extent of the paranasal sinus mass. (A) Coronal T1-weighted MRI with contrast demonstrating right orbital displacement without radiologic evidence of periorbital invasion. (B) Sagittal T1-weighted MRI with contrast demonstrating anterior-posterior tumor limits and intracranial extension. 


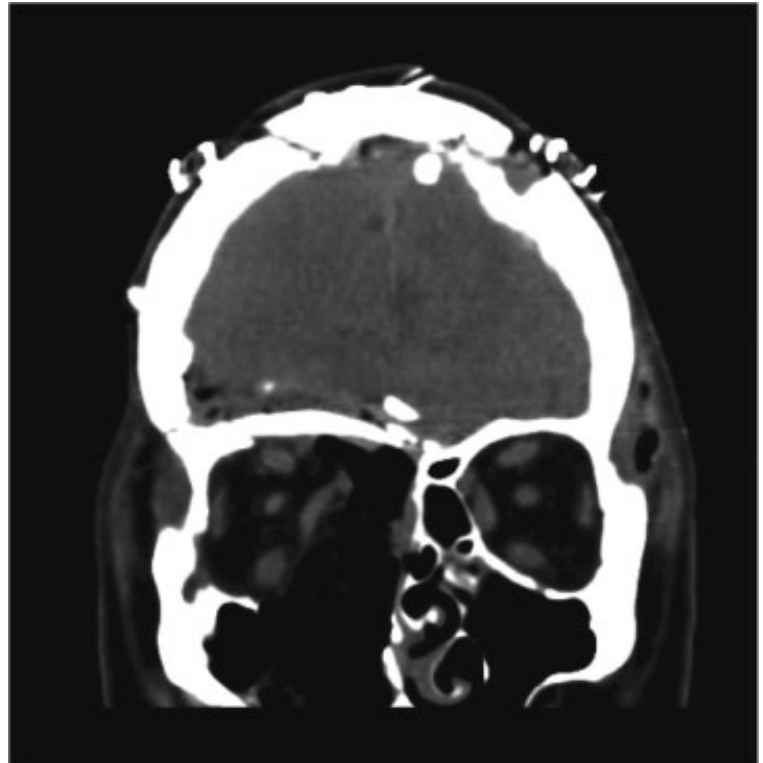

Fig. 2 Postoperative coronal computed tomography demonstrating resection of the tumor and orbital roof reconstruction.

There was no clinical or radiologic evidence of tumor recurrence 12 months postresection.

Final pathologic examination confirmed the diagnosis of cellular schwannoma. Microscopic examination revealed a nonencapsulated, highly cellular spindle cell lesion infiltrating the sinonasal submucosa and demonstrating bone invasion. No significant atypia was identified. There was no necrosis and the mitotic rate was quite low, in comparison with the cellularity of the lesion, with approximately one to two mitoses per 10 high-power fields. The lesion stained strongly and diffusely positive for S100, and Ki-67 (proliferation index marker) highlighted $6.7 \%$ of tumor cells ( 1,000 cells counted) (-Fig. 3 ).

\section{Discussion}

Head and neck schwannomas rarely occur in the sinonasal cavities, and they are typically described as solitary encapsulated lesions confined to the nasoethmoid region. ${ }^{1-5,13-17}$ The less common cellular schwannoma has been described in the sinonasal tract, and the lack of encapsulation along with bony erosion of this variant was previously noted. ${ }^{11,16}$ Although schwannomas are uncommon in this location, and cellular schwannomas even more so, the recognition of this entity in this anatomical site is important. ${ }^{1-5,13-17}$

The reported case reiterates the potential risk of rendering a malignant diagnosis on biopsy, particularly in light of the aggressive radiographic findings. Although bone erosion is well described in cellular schwannomas at a variety of anatomical locations, it appears that those arising in this anatomical site tend to have the additional aggressive feature of being nonencapsulated with an infiltrative growth pattern. These findings, along with the rarity of this lesion in this location, increase the likelihood that a malignant diagnosis may be rendered.

This case was a diagnostic challenge, which was demonstrated by a difference in initial biopsy pathology and postexcision pathology findings. Initial biopsies revealed a spindle

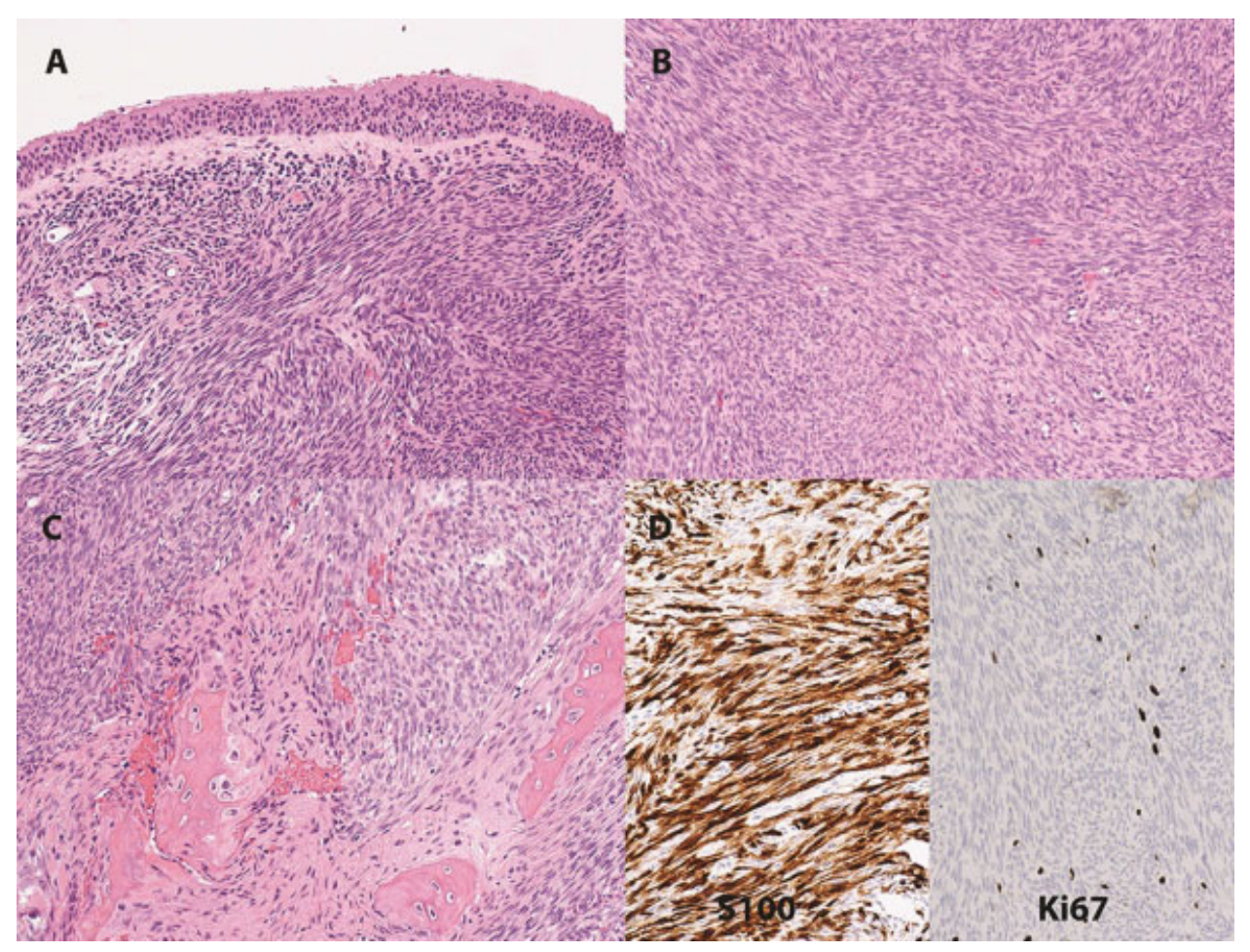

Fig. 3 (A) Low-power view $(\times 40)$ of a highly cellular spindle cell lesion arranged in sweeping fascicles, infiltrating and expanding the submucosa with intact overlying sinonasal mucosa. (B) Medium-power view demonstrating long sweeping fascicles of uniform spindle cells. (C) Tumor with embedded bony spicules due to erosion/infiltration into adjacent bone. (D) (left) Tumor cells demonstrate strong, diffuse S100 immunoreactivity; (right) Ki-67 highlighted $6.7 \%$ of tumor cells (1,000 cells counted), and only one to two mitoses per 10 high-power fields were identified. 
Table 1 Microscopic and immunohistochemical features to distinguish among conventional schwannoma, cellular schwannoma, and malignant peripheral nerve sheath tumor

\begin{tabular}{|l|l|l|l|}
\hline & Conventional schwannoma \\
Microscopic & $\begin{array}{l}\text { Antoni A (hypercellular) and } \\
\text { Antoni B (loose/hypocellular) } \\
\text { areas; thick-walled hyalinized } \\
\text { blood vessels; no/rare mitoses }\end{array}$ & $\begin{array}{l}\text { Cellular schwannoma } \\
\text { areas; cells may be hyperchro- } \\
\text { matic with or without pleomor- } \\
\text { phic; thick-walled hyalinized } \\
\text { blood vessels; mitoses typically } \\
\leq 4 \text { per 10 high-power fields }\end{array}$ & $\begin{array}{l}\text { Markedly hypercellular spindle } \\
\text { ofls in fascicular pattern, cells } \\
\text { chromatic; geographic necro- } \\
\text { sis and mitoses }>4 \text { per } 10 \\
\text { high-power fields; some may } \\
\text { have epithelioid cells and some } \\
\text { may have heterologous } \\
\text { elements }\end{array}$ \\
\hline S100 immunostaining & Strong diffuse staining & Strong diffuse staining & $\begin{array}{l}\text { Scattered positive cells in } ~ \\
50-70 \% \text { of cases; can be } \\
\text { strongly positive in epithelioid } \\
\text { variant of MPNST }\end{array}$ \\
\hline
\end{tabular}

Abbreviation: MPNST, malignant peripheral nerve sheath tumor.

cell neoplasm that was suspicious for malignancy due to the lack of encapsulation, the infiltration of the sinonasal submucosa, and tumor invasion into bone. Although these findings were still present in the final postexcision specimen, the lack of malignant histologic features, such as necrosis, mitoses, and significant atypia, along with strong, diffuse S100 immunoreactivity confirmed the diagnosis of an unencapsulated cellular schwannoma.

Of particular significance in this case was the presence of bone infiltration and erosion with subsequent intracranial tumor extension. Bone erosion has been described in cellular schwannoma but typically as a pushing front into the adjacent bone. ${ }^{10}$ In this case, the tumor appeared to infiltrate the adjacent bone more aggressively, with bone spicules present within the tumor mass. There are very few reported cases of sinonasal cellular schwannoma with intracranial extension, and thus our case is an important addition to the literature.

Classic schwannomas typically contain a capsule believed to be derived from perineurium of the native nerve. ${ }^{3}$ Although there are many possibilities for the nerve origin for sinonasal schwannomas, this cellular schwannoma likely originated from sinonasal mucosal autonomic nerves because these nerves lack a perineural layer. ${ }^{2,3,15}$ The nonencapsulated nature of this tumor accounted for its more infiltrative and aggressive nature.

Furthermore, given the rarity of this pathology, it is important to differentiate correctly between a conventional and a cellular schwannoma when reporting in the literature. This will allow us to better understand the clinical behavior and treatment results from the management of this pathology in the future. Histologic criteria to differentiate schwannoma, cellular schwannoma, and malignant peripheral nerve sheath tumor (MPNST) were previously described and are summarized in - Table 1. ${ }^{11}$ Using these criteria, we identified one publication with misclassified tumors. ${ }^{3}$ The histologic findings listed in Mey et al suggest that cases 1 to 4 are cellular schwannomas rather than conventional schwannomas based on the predominance of the Antoni A pattern, little to no Antoni B pattern, and mitoses and/or increased Ki-67. ${ }^{3}$ Case number 5 in the same article labeled as a malignant transfor- mation of a schwannoma, is most likely an epithelioid MPNST, based on the decreased S100 staining (compared with cases 1-4) and atypical areas identified in -Fig. 2 in both the primary and the recurrence. The distinction between conventional schwannomas versus cellular schwannomas, which may have malignant clinical features, is more clearly distinguished in the pathology literature. For instance, cellular schwannoma involving the paranasal sinuses was actually first described in the publication by Hasegawa et al and not by al-Otieschan et al. ${ }^{11,16}$ Given the difference in clinical behavior between these two pathologies, close collaboration with a pathologist would be helpful for correctly reporting cases of schwannoma in the future.

The reported patient received postoperative externalbeam radiation therapy given the close intracranial resection margins. The treatment of a conventional schwannoma is typically surgical excision. ${ }^{1}$ However, given the aggressive and diffusely infiltrative nature of the cellular schwannoma reported in this case (which differentiates it from conventional schwannoma), adjuvant radiotherapy was administered. The use of adjuvant radiotherapy was reported in the literature for other patients with cellular schwannoma but with numbers too small to provide data on efficacy. ${ }^{10}$

\section{Conclusion}

This case of sinonasal cellular schwannoma is important because its clinicopathologic features are easily mistaken for a malignant entity. It should be emphasized that with this pathology, patient presentation may be variable and that biopsy and imaging alone do not fully outline the invasive potential of these tumors. Therefore, we suggest that sinonasal cellular schwannoma be considered in the differential diagnosis of a poorly defined invasive paranasal sinus mass.

\section{Note}

This material has never been published and is not currently under evaluation in any other peer-reviewed publication. 


\section{References}

1 Suh JD, Ramakrishnan VR, Zhang PJ, et al. Diagnosis and endoscopic management of sinonasal schwannomas. ORL J Otorhinolaryngol Relat Spec 2011;73(6):308-312

2 Yu E, Mikulis D, Nag S. CT and MR imaging findings in sinonasal schwannoma. AJNR Am J Neuroradiol 2006;27(4):929-930

3 Mey KH, Buchwald C, Daugaard S, Prause JU. Sinonasal schwannoma-a clinicopathological analysis of five rare cases. Rhinology 2006;44(1):46-52

4 Hu J, Bao YY, Cheng KJ, Zhou SH, Ruan LX, Zheng ZJ. Computed tomography and pathological findings of five nasal neurilemmomas. Head Neck Oncol 2012;4:26

5 Kim YS, Kim HJ, Kim CH, Kim J. CT and MR imaging findings of sinonasal schwannoma: a review of 12 cases. AJNR Am J Neuroradiol 2013;34(3):628-633

6 Jacopo G, Micaela I, Italo C, Luigi C, Larocca LM, Gaetano P. Atypical sinonasal schwannomas: a difficult diagnostic challenge. Auris Nasus Larynx 2009;36(4):482-486

7 Woodruff JM, Godwin TA, Erlandson RA, Susin M, Martini N. Cellular schwannoma: a variety of schwannoma sometimes mistaken for a malignant tumor. Am J Surg Pathol 1981;5(8):733744

8 Fletcher CD, Davies SE, McKee PH. Cellular schwannoma: a distinct pseudosarcomatous entity. Histopathology 1987;11(1): 21-35
9 Lodding P, Kindblom LG, Angervall L, Stenman G. Cellular schwannoma. A clinicopathologic study of 29 cases. Virchows Arch A Pathol Anat Histopathol 1990;416(3):237-248

10 White W, Shiu MH, Rosenblum MK, Erlandson RA, Woodruff JM. Cellular schwannoma. A clinicopathologic study of 57 patients and 58 tumors. Cancer 1990;66(6):1266-1275

11 Hasegawa SL, Mentzel T, Fletcher CD. Schwannomas of the sinonasal tract and nasopharynx. Mod Pathol 1997;10(8):777-784

12 Casadei GP, Scheithauer BW, Hirose T, Manfrini M, Van Houton C, Wood MB. Cellular schwannoma. A clinicopathologic, DNA flow cytometric, and proliferation marker study of 70 patients. Cancer 1995;75(5):1109-1119

13 Nagayama I, Nishimura T, Furukawa M. Malignant schwannoma arising in a paranasal sinus. J Laryngol Otol 1993;107(2):146-148

14 George KJ, Price R. Nasoethmoid schwannoma with intracranial extension. Case report and review of literature. $\mathrm{Br} \mathrm{J}$ Neurosurg 2009;23(1):83-85

15 Maheshwari GK, Baboo HA, Gopal U, Baiar DB, Shah NM. Malignant schwannoma of the sinonasal tract. Indian J Otolaryngol Head Neck Surg 1999;51(1):47-50

16 al-Otieschan AT, Manohar MB, Gangopadhyay K, Tulbah A. Cellular schwannoma of the paranasal sinuses: initial report of a case. Ear Nose Throat J 2002;81(4):281-284

17 Yang TL, Hsu MC, Liu CM. Nasal schwannoma: a case report and clinicopathologic analysis. Rhinology 2001;39(3):169-172 